\title{
Non-resonant dynamic stark control of vibrational motion with optimized laser pulses
}

\author{
Thomas, Esben Folger; Henriksen, Niels Engholm
}

Published in:

Journal of Chemical Physics

Link to article, DOI:

$10.1063 / 1.4954663$

Publication date:

2016

Document Version

Publisher's PDF, also known as Version of record

Link back to DTU Orbit

Citation (APA):

Thomas, E. F., \& Henriksen, N. E. (2016). Non-resonant dynamic stark control of vibrational motion with optimized laser pulses. Journal of Chemical Physics, 144, [244307]. https://doi.org/10.1063/1.4954663

\section{General rights}

Copyright and moral rights for the publications made accessible in the public portal are retained by the authors and/or other copyright owners and it is a condition of accessing publications that users recognise and abide by the legal requirements associated with these rights.

- Users may download and print one copy of any publication from the public portal for the purpose of private study or research.

- You may not further distribute the material or use it for any profit-making activity or commercial gain

- You may freely distribute the URL identifying the publication in the public portal

If you believe that this document breaches copyright please contact us providing details, and we will remove access to the work immediately and investigate your claim 


\section{A|P| $\begin{aligned} & \text { The Journal of } \\ & \text { Chemical Physics }\end{aligned}$}

\section{Non-resonant dynamic stark control of vibrational motion with optimized laser pulses}

Esben F. Thomas and Niels E. Henriksen

Citation: The Journal of Chemical Physics 144, 244307 (2016); doi: 10.1063/1.4954663

View online: http://dx.doi.org/10.1063/1.4954663

View Table of Contents: http://scitation.aip.org/content/aip/journal/jcp/144/24?ver=pdfcov

Published by the AIP Publishing

\section{Articles you may be interested in}

Laser control of the radiationless decay in pyrazine using the dynamic Stark effect

J. Chem. Phys. 140, 194309 (2014); 10.1063/1.4875736

Stark-induced adiabatic Raman passage for preparing polarized molecules

J. Chem. Phys. 135, 024201 (2011); 10.1063/1.3599711

Electronic-resonance-enhanced coherent anti-Stokes Raman scattering of nitric oxide: Saturation and Stark effects

J. Chem. Phys. 133, 084310 (2010); 10.1063/1.3474702

Pump-dump iterative squeezing of vibrational wave packets

J. Chem. Phys. 123, 244101 (2005); 10.1063/1.2139091

Photodissociation detection of microwave transitions in highly excited vibrational states

J. Chem. Phys. 111, 3488 (1999); 10.1063/1.479633

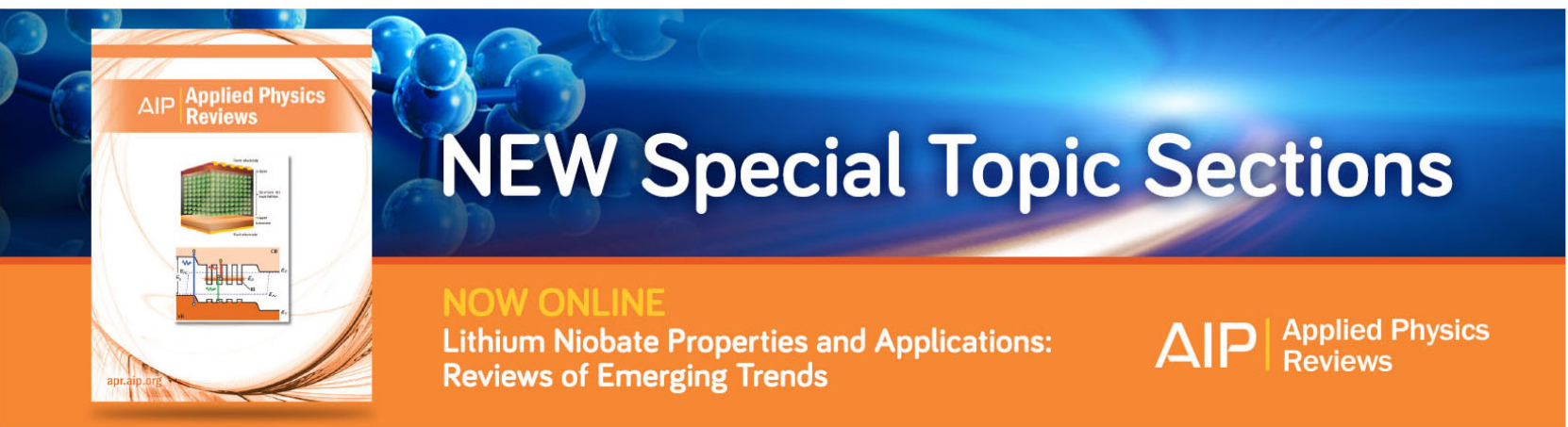




\title{
Non-resonant dynamic stark control of vibrational motion with optimized laser pulses
}

\author{
Esben F. Thomas and Niels E. Henriksen \\ Department of Chemistry, Technical University of Denmark, Building 207, DK-2800 Kongens Lyngby, Denmark
}

(Received 2 May 2016; accepted 10 June 2016; published online 28 June 2016)

\begin{abstract}
The term dynamic Stark control (DSC) has been used to describe methods of quantum control related to the dynamic Stark effect, i.e., a time-dependent distortion of energy levels. Here, we employ analytical models that present clear and concise interpretations of the principles behind DSC. Within a linearly forced harmonic oscillator model of vibrational excitation, we show how the vibrational amplitude is related to the pulse envelope, and independent of the carrier frequency of the laser pulse, in the DSC regime. Furthermore, we shed light on the DSC regarding the construction of optimal pulse envelopes - from a time-domain as well as a frequency-domain perspective. Finally, in a numerical study beyond the linearly forced harmonic oscillator model, we show that a pulse envelope can be constructed such that a vibrational excitation into a specific excited vibrational eigenstate is accomplished. The pulse envelope is constructed such that high intensities are avoided in order to eliminate the process of ionization. Published by AIP Publishing. [http://dx.doi.org/10.1063/1.4954663]
\end{abstract}

\section{INTRODUCTION}

Since the advent of the laser in the 1960's, the possibility of using coherent light sources to control molecules and molecular reactions has been a hot topic in photochemistry. ${ }^{1-6}$ The ability to generate laser pulses on ever shorter time scales, down to femto- and even atto-second durations, as well as the development of optical techniques for actively controlling the time-dependent features of these pulses, has driven the field forward into new lines of theoretical and experimental inquiry. In general, the attainment of tools that are controllable on the same characteristic time scales as the molecular systems on which they act has led to a deeper understanding of a variety of time-dependent quantum phenomena.

Experimentally, capabilities have advanced in step with developments in laser, computer, and optical technology. In 1992 Judson and Rabitz ${ }^{7}$ described a novel method of combining adjustable spectral pulse shapers ${ }^{8,9}$ with evolutionary algorithms ${ }^{10}$ in closed-loop schemes that could generate shaped laser pulses for driving molecular systems into desired states. While this technique has been demonstrated and employed successfully in a number of experimental applications, ${ }^{5,11-16}$ the complexity of the systems, and of the pulses that are generated via this kind of "black box" optimization approach, makes it difficult to ascertain the mechanisms behind the interaction dynamics.

At higher field strengths, polarization forces, i.e., the distortion of electronic states leading to induced dipole moments related to the polarizability, can play an important role. The application and control of these polarization forces (via ultrashort optimized laser pulses) has been exploited experimentally in order to control the rotational, vibrational, and dissociation dynamics of molecules. The application of laser pulses with a temporal duration shorter than a rotational period leads to post-pulse time-dependent molecular alignment due to the sudden switch-on/-off of an angular-dependent interaction potential. ${ }^{17}$ Similarly, time-dependent modifications of the interaction potential associated with vibrational/torsional motion in molecules have been reported. ${ }^{18-20}$ For non-adiabatic motion in molecular photodissociation involving excited electronic states, similar effects have been used to modify the dissociation dynamics. ${ }^{21-24} \mathrm{~A}$ key feature of the above phenomena involving polarization forces is that control can be exerted under non-resonant conditions.

The challenge for theorists is to develop models and techniques that describe the interaction between an electromagnetic field and a molecule that are accurate enough to be realistic, yet simple enough to be understood qualitatively. A few theoretical studies have discussed the systematic numerical treatment of polarization effects, ${ }^{25,26}$ and some approaches have treated the interaction approximately; ${ }^{27-30}$ however, the majority of theoretical studies in laser control of chemical dynamics have only included permanent dipole moments in the laser-molecule interaction.

The motivation behind this paper is to enhance our physical insight into time-dependent shifts in relevant electronic energy levels that can arise due to polarization forces. When a molecule interacts with a time-dependent electric field, the distortion of the electronic states leads to the so-called dynamic Stark effect, ${ }^{22,31}$ which as the name suggests is the time-dependent counterpart to the static Stark effect. $^{32}$ The term dynamic Stark control (DSC) has been used to describe methods of quantum control related to the dynamic Stark effect (see, e.g., Refs. 18 and 21). At this point, it is also pertinent to mention the related phenomenon of Impulsive Stimulated Raman Scattering (ISRS). ${ }^{33-37}$ One of our intentions here is to employ analytical models that present clear and concise interpretations of the principles behind DSC, so that we may develop our intuitive understanding of how these effects operate. 
The structure and main results of this paper are summarized in the following. For completeness, we start with a derivation of the laser-molecule interaction within the BornOppenheimer approximation, including the polarization term described in a perturbative manner. Then, within a linearly forced harmonic oscillator model of vibrational excitation, we show analytically (in the non-resonant regime) how the vibrational amplitude is related to the pulse envelope and independent of the carrier frequency of the laser pulse. Furthermore, we shed light on the DSC regarding the construction of optimal pulse envelopes - from a time-domain as well as a frequency-domain perspective.

Finally, in a numerical study beyond the linearly forced harmonic oscillator model, we show that a pulse envelope can be constructed such that a vibrational excitation into a specific excited vibrational eigenstate is accomplished. The pulse envelope is constructed such that high intensities are avoided in order to eliminate the process of ionization. Since control can be exerted under non-resonant conditions, we show that ignoring the carrier frequency allows for a relaxation of the criteria that an optimized pulse must fulfill, which in turn may help facilitate experimental implementation of theoretical results.

\section{MODELING THE FIELD/MOLECULE INTERACTION}

The general Hamiltonian of a molecule can be written as $\hat{H}=\hat{T}_{\text {nuc }}+\hat{H}_{e}$, where $\hat{T}_{\text {nuc }}$ is the nuclear kinetic energy and $\hat{H}_{e}$ is the electronic Hamiltonian. Invoking the adiabatic approximation, and confining ourselves to the electronic ground state $\psi_{0}$, we can calculate the electronic energy as a function of the nuclear position $\mathbf{R}$ by solving the equation

$$
\hat{H}_{e} \psi_{0}(\mathbf{R} ; \mathbf{r})=E_{0}(\mathbf{R}) \psi_{0}(\mathbf{R} ; \mathbf{r}),
$$

where $\mathbf{R}$ and $\mathbf{r}$ denote the respective nuclear and electronic position vectors, $\mathbf{R} ; \mathbf{r}$ denotes fixed $\mathbf{R}$ coordinates, and $E_{0}(\mathbf{R})$ is the electronic energy. Within the Born-Oppenheimer approximation, the time-dependent Schrödinger equation for the nuclear wave function $\chi_{0}(\mathbf{R}, t)$ will take the form

$$
i \hbar \frac{\partial}{\partial t} \chi_{0}(\mathbf{R}, t)=\left[\hat{T}_{\mathrm{nuc}}+E_{0}(\mathbf{R})\right] \chi_{0}(\mathbf{R}, t) .
$$

When interacting with a molecule, a coherent laser pulse can be approximated as a spatially homogenous, time-dependent electric field $\boldsymbol{\varepsilon}(t)$, provided the carrier wavelength is large compared to the molecular dimensions. In this limit, the interaction of the field with the electrons and nuclei within the electric-dipole approximation can be written as the following contribution to the molecular Hamiltonian,

$$
\hat{H}_{\text {int }}=-\boldsymbol{\mu} \cdot \boldsymbol{\varepsilon}(t),
$$

where $\boldsymbol{\mu}$ is the electric dipole moment. When this term is introduced into Eq. (1), the field-free potential energy surface $E_{0}(\mathbf{R})$ will become modified due to the interaction with $\boldsymbol{\varepsilon}(t)$. This modified energy surface, which we will call $E_{\text {int }}$ (we will simplify the notation in the following steps by omitting functional dependencies on $\mathbf{R}$ and $\mathbf{r}$ ), can be expressed as

$$
E_{\text {int }}=\left\langle\psi_{0}^{\varepsilon}\left|\hat{H}_{e}-\boldsymbol{\mu} \cdot \boldsymbol{\varepsilon}(t)\right| \psi_{0}^{\varepsilon}\right\rangle,
$$

where the ${ }^{\varepsilon}$ superscript on $\psi_{0}^{\varepsilon}$ indicates that the electronic wave function has been modified by the external field, and the integration is over the electronic coordinates $\mathbf{r}$. As stated, some treatments correct for the shift in charge distribution by explicitly calculating the $\psi_{0}^{\varepsilon}$ eigenfunctions in the presence of external fields of varying strengths. ${ }^{25}$ While this yields a solution that is in principle exact, the complexity of the approach can obfuscate the dynamics behind the interaction. We will instead utilize a simpler approach that treats the influence of the field on the electronic wave functions as a perturbation. We simplify the subsequent analysis by aligning the polarization direction of the external field with the $z$ axis, i.e., $\boldsymbol{\varepsilon}(t)=\varepsilon(t) \hat{z}$, and $\boldsymbol{\mu}=\mu_{z}$. Assuming that $\varepsilon(t)$ is sufficiently small, we can express the modified potential energy surface $E_{\text {int }}(\mathbf{R})$ as a second-order Taylor expansion around the unperturbed potential function $E_{0}(\mathbf{R})$,

$$
E_{\mathrm{int}} \approx E_{0}+\left(\frac{d E_{\mathrm{int}}}{d \varepsilon(t)}\right)_{0} \varepsilon(t)+\frac{1}{2}\left(\frac{d^{2} E_{\mathrm{int}}}{d \varepsilon^{2}(t)}\right)_{0} \varepsilon^{2}(t),
$$

where the ${ }_{0}$ subscript indicates that the derivative is evaluated at $\varepsilon(t)=0$. Applying the Hellmann-Feynman theorem ${ }^{38}$ to Eq. (4), it can be shown that differentiation of $E_{\text {int }}$ with respect to $\varepsilon(t)$ yields

$$
\frac{d E_{\text {int }}}{d \varepsilon(t)}=-\left\langle\mu_{z}\right\rangle
$$

Combining Eqs. (5) and (6) leads to the relation

$$
\left\langle\mu_{z}\right\rangle=-\left(\frac{d E_{\text {int }}}{d \varepsilon(t)}\right)_{0}-\left(\frac{d^{2} E_{\text {int }}}{d \varepsilon^{2}(t)}\right)_{0} \varepsilon(t) .
$$

We can derive explicit functions for these two terms by applying first-order perturbation theory to the electronic wave functions and assuming that the envelope of $\varepsilon(t)$ changes slowly relative to the characteristic time scales of the system, i.e., we disregard any transient oscillations in electronic density that may arise due to the sudden application of a perturbing field, ${ }^{27}$

$$
\left\langle\mu_{z}\right\rangle=\left\langle\psi_{0}\left|\mu_{z}\right| \psi_{0}\right\rangle+\frac{2}{\hbar} \sum_{n \neq 0} \frac{\omega_{n 0}\left|\left\langle\psi_{n}\left|\mu_{z}\right| \psi_{0}\right\rangle\right|^{2}}{\omega_{n 0}^{2}-\omega_{c}^{2}} \varepsilon(t),
$$

where $\omega_{n 0}=\left(E_{n}-E_{0}\right) / \hbar$ are the unperturbed electronic state transition frequencies, $\omega_{c}$ is the carrier frequency of $\varepsilon(t)$, and the $n \neq 0$ subscript indicates that the ground state is omitted from the sum. In our analysis, we assume that $\omega_{c} \ll \omega_{n 0}$, i.e., the field carrier frequency is much lower than the electronic state transition frequencies of the molecule, which is generally true as long as the nuclear motion is confined to the electronic ground state. In this case Eq. (8) simplifies to

$$
\left\langle\mu_{z}\right\rangle=\left\langle\psi_{0}\left|\mu_{z}\right| \psi_{0}\right\rangle+\frac{2}{\hbar} \sum_{n \neq 0} \frac{\left|\left\langle\psi_{n}\left|\mu_{z}\right| \psi_{0}\right\rangle\right|^{2}}{\omega_{n 0}} \varepsilon(t) .
$$

The first and second terms on the right hand side of Eq. (9) are the permanent electric dipole (PED) and static molecular polarizability (SMP) terms, respectively. The PED term is merely the charge distribution of the field-free molecule. If the field is relatively weak, the molecular electrons will not be significantly perturbed and the PED term is sufficient to accurately describe the dynamics of the system. If the 
molecule is subjected to more intense fields, the $\psi_{0}$ electronic wave functions will be significantly modified, leading to a shift in the molecular charge distribution that the PED will not be able to account for. The SMP term is the first order correction to this field induced charge shift and is therefore linearly proportional to the field amplitude $\varepsilon(t)$.

It is possible to extend the aforementioned principle of derivation to include higher orders of $\varepsilon(t)$. Nevertheless, in this paper it will be shown that even when only the PED and SMP terms are included, it is possible to qualitatively reproduce many of the behaviors observed in more complicated models, the strength of the approach being that we are afforded the clarity that comes from being able to derive relatively simple analytical solutions for the time-dependent dynamics.

While Eq. (9) demonstrates how the PED and SMP terms may be calculated provided the electronic eigenfunctions are known, we will now disregard the exact form of these functions and instead focus on their relation to $\varepsilon(t)$. Equation (9) can thus be recast in a simplified form

$$
\left\langle\mu_{z}\right\rangle=\mu+\alpha \varepsilon(t)
$$

Comparing Eqs. (10), (7), and (5), we see that

$$
E_{\text {int }}=E_{0}-\mu \varepsilon(t)-\frac{1}{2} \alpha \varepsilon^{2}(t) .
$$

Within the Born-Oppenheimer approximation, $\mu$ and $\alpha$ will be functions of $\mathbf{R}$ as they contain the electronic eigenfunctions $\psi_{n}(\mathbf{R} ; \mathbf{r})$, where the electronic coordinates have been integrated out in the matrix elements of Eq. (9). Returning to Eq. (2), we can now update the expression to include the second order correction to the energy that arises due to coupling with the external field, reinstating the functional dependencies on $\mathbf{R}$ for completeness,

$i \hbar \frac{\partial}{\partial t} \chi_{0}(\mathbf{R}, t)=\left[\hat{T}_{\text {nuc }}+E_{0}(\mathbf{R})-\hat{C}_{\text {int }}(\mathbf{R}, t)\right] \chi_{0}(\mathbf{R}, t)$

where

$$
\hat{C}_{\text {int }}(\mathbf{R}, t)=\mu(\mathbf{R}) \varepsilon(t)+\frac{1}{2} \alpha(\mathbf{R}) \varepsilon^{2}(t) .
$$

\section{A LINEARLY FORCED HARMONIC OSCILLATOR MODEL OF DSC}

In order to gain insight into the wave packet dynamics described by Eqs. (12) and (13), we consider a onedimensional model of a simple diatomic molecule aligned with the field polarization direction, with interatomic distance coordinate $R$. If we assume that the variation in $R$ is small, we can approximate $\mu(R)$ and $\alpha(R)$ as linear expansions around the molecular equilibrium distance $R_{\text {eq. }}$. It can be shown that the zeroth order terms of these expansions will not impact the dynamics of the field/molecule interaction, and can therefore be disregarded. ${ }^{39}$ Approximating the unperturbed ground state energy surface as a harmonic expansion around $R_{\text {eq }}$, the potential energy of the molecule takes the form of a linearly forced harmonic oscillator with eigenfrequency $\omega_{0}$,

$$
\begin{aligned}
V(R, t)= & \frac{1}{2} m \omega_{0}^{2} R^{2} \\
& -\left[\left(\frac{d \mu(R)}{d R}\right)_{\mathrm{eq}} \varepsilon(t)+\frac{1}{2}\left(\frac{d \alpha(R)}{d R}\right)_{\mathrm{eq}} \varepsilon^{2}(t)\right] R,
\end{aligned}
$$

where we have set $R_{\text {eq }}=0$ to simplify the notation, and the eq Subscript indicates that the derivative is taken at $R=R_{\mathrm{eq}}=0$. We denote the expectation value of the wave packet position at time $t$ as $\langle R\rangle(t)$. Ehrenfest's theorem can be used to show that the expectation value in a harmonic potential will obey classical dynamics. ${ }^{40}$ This allows us simplify the description of the wave packet by letting $\langle R\rangle(t) \equiv R(t)$, where $R(t)$ denotes the position of a classical point particle. Assuming $R\left(t_{0}\right)=0$, the position expectation value of the wave packet at time $t$ can be found by solving the integral, ${ }^{41}$

$$
\begin{aligned}
R(t)= & \frac{1}{\omega_{0} m}\left[\sin \left(\omega_{0} t\right) \int_{t_{0}}^{t} \cos \left(\omega_{0} t\right) F(t) d t\right. \\
& \left.-\cos \left(\omega_{0} t\right) \int_{t_{0}}^{t} \sin \left(\omega_{0} t\right) F(t) d t\right],
\end{aligned}
$$

where $F(t)$ represents the time-dependent component of the differential equation, which in our case can be found from Eq. (14) to be

$$
F(t)=\left[\mu^{\prime} \varepsilon(t)+\frac{1}{2} \alpha^{\prime} \varepsilon^{2}(t)\right],
$$

where we recast $\alpha^{\prime}=(d \alpha(R) / d R)_{\mathrm{eq}}$ and $\mu^{\prime}=(d \mu(R) / d R)_{\mathrm{eq}}$ to simplify the notation. Inserting $F(t)$ into Equation (15), we can split the resulting expression into two components $R(t)=R_{\mu}(t)+R_{\alpha}(t)$, where

$$
\begin{aligned}
R_{\mu}(t)= & \frac{\mu^{\prime}}{\omega_{0} m}\left[\sin \left(\omega_{0} t\right) \int_{t_{0}}^{t} \cos \left(\omega_{0} t\right) \varepsilon(t) d t\right. \\
& \left.-\cos \left(\omega_{0} t\right) \int_{t_{0}}^{t} \sin \left(\omega_{0} t\right) \varepsilon(t) d t\right]
\end{aligned}
$$

and

$$
\begin{aligned}
R_{\alpha}(t)= & \frac{\alpha^{\prime}}{2 \omega_{0} m}\left[\sin \left(\omega_{0} t\right) \int_{t_{0}}^{t} \cos \left(\omega_{0} t\right) \varepsilon^{2}(t) d t\right. \\
& \left.-\cos \left(\omega_{0} t\right) \int_{t_{0}}^{t} \sin \left(\omega_{0} t\right) \varepsilon^{2}(t) d t\right] .
\end{aligned}
$$

Now, let $\varepsilon(t)$ take the form of a transform limited Gaussian pulse with amplitude $A_{0}$, second moment pulse width $\sigma$ and (positive) angular carrier frequency $\omega_{c}$,

$$
\varepsilon(t)=A_{0} \cos \left(\omega_{c} t\right) \exp \left(-\frac{t^{2}}{2 \sigma^{2}}\right),
$$

where it is assumed that $\omega_{c} \sigma \gg 1$, i.e., the pulse envelope is wide enough to allow for multiple oscillations. When this pulse interacts with the system, it may induce oscillations in the position of the wave packet which will continue after the pulse has died out. Combining Eqs. (17)-(19), and evaluating the integrals in the limit where $t_{0} \rightarrow-\infty$ and $t \rightarrow \infty$, the parity of the integrand functions leads to the elimination of the second terms in the brackets of Eqs. (17) and (18). The amplitude of the wave packet oscillation in the $t \rightarrow \infty$ limit, 
which will henceforth be denoted by $\Gamma$, can be written as

$\Gamma\left(A_{0}, \omega_{c}, \sigma\right)=\left(I_{\mu}^{2}\left(A_{0}, \omega_{c}, \sigma\right)+I_{\alpha}^{2}\left(A_{0}, \omega_{c}, \sigma\right)\right)^{1 / 2}$,

where

$$
\begin{aligned}
I_{\mu}\left(A_{0}, \omega_{c}, \sigma\right)= & \frac{\mu^{\prime} A_{0} \sigma}{\omega_{0} m} \sqrt{\frac{\pi}{2}}\left[\exp \left(-\frac{\sigma^{2}\left(\omega_{0}-\omega_{c}\right)^{2}}{2}\right)\right. \\
& \left.+\exp \left(-\frac{\sigma^{2}\left(\omega_{0}+\omega_{c}\right)^{2}}{2}\right)\right]
\end{aligned}
$$

and

$$
\begin{aligned}
I_{\alpha}\left(A_{0}, \omega_{c}, \sigma\right)= & \frac{\alpha^{\prime} A_{0}^{2} \sigma \sqrt{\pi}}{4 \omega_{0} m}\left[\exp \left(-\frac{\sigma^{2} \omega_{0}^{2}}{4}\right)\right. \\
& +\frac{1}{2} \exp \left(-\frac{\sigma^{2}\left(\omega_{0}-2 \omega_{c}\right)^{2}}{4}\right) \\
& \left.+\frac{1}{2} \exp \left(-\frac{\sigma^{2}\left(\omega_{0}+2 \omega_{c}\right)^{2}}{4}\right)\right],
\end{aligned}
$$

where $\Gamma\left(A_{0}, \omega_{c}, \sigma\right)$ indicates that the wave packet amplitude is dependent on the pulse amplitude, carrier frequency, and width and $I_{\mu}\left(A_{0}, \omega_{c}, \sigma\right)$ and $I_{\alpha}\left(A_{0}, \omega_{c}, \sigma\right)$ are the contributions from the dipole and polarizability terms, respectively. Inspecting Eq. (21), it is clear that the contribution from the first bracketed term becomes large when the carrier frequency $\omega_{c}$ is close to the harmonic eigenfrequency $\omega_{0}$. Likewise, the middle term in Eq. (22) will become large when $\omega_{c} \approx \frac{1}{2} \omega_{0}$. We note in passing that this result is similar to one obtained in a study in which the excitation of a homonuclear diatomic molecule was investigated using a complete quantum mechanical treatment. ${ }^{25}$ The two aforementioned contributions to the wave packet amplitude can be interpreted as one and two photon absorption processes, respectively. These terms are dependent on the pulse carrier frequency, and their behavior differs qualitatively from the first term in the brackets on the right side of Eq. (22), which dominates in the $\left|\omega_{c}\right| \gg \omega_{0}$ limit, in which Eq. (20) is reduced to the following form:

$$
\Gamma\left(A_{0}, \sigma\right)_{\left|\omega_{c}\right| \gg \omega_{0}}=\frac{\alpha^{\prime} A_{0}^{2} \sigma \sqrt{\pi}}{4 \omega_{0} m} \exp \left(-\frac{\sigma^{2} \omega_{0}^{2}}{4}\right) .
$$

In this limit, we see the behavior that characterizes the dynamic Stark effect, since the wave packet amplitude in this regime is no longer influenced by the pulse carrier frequency. To further illustrate this concept, a graphical representation of $\Gamma\left(A_{0}, \omega_{c}, \sigma\right)$ for parameters selected to approximate the $\mathrm{HCl}$ electronic ground state $\mathrm{s}^{42,43}$ is shown in Fig. 1. Here, the function is plotted for varying values of $\sigma$ and $\omega_{c}$, at a constant peak pulse intensity of $10 \mathrm{TW} / \mathrm{cm}^{2}$. The two horizontal excitation "channels" visible in the top half of the figure arise due to the one and two photon absorption terms. The bottom half of the figure shows how the wave packet response becomes independent of carrier frequency as the system parameters move into the dynamic Stark regime.

Note also how the wave packet amplitude in Fig. 1 starts to primarily respond to changes in the pulse width when $\left|\omega_{c}\right| \gg \omega_{0}$, as Eq. (23) indicates. Fig. 2 shows a 1D plot of variations in $\Gamma$ as a function of the pulse width in the region under the red line in Fig. 1.

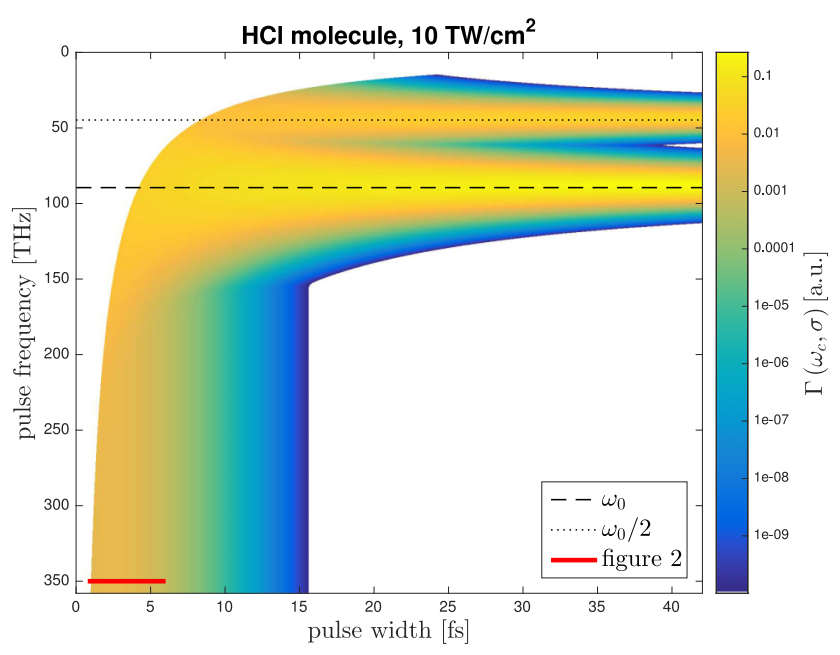

FIG. 1. The wave packet oscillation amplitude $\Gamma\left(\omega_{c}, \sigma\right)$ (Eq. (20)) as a function of pulse carrier frequency $\omega_{c}$ and pulse width $\sigma$. For comparison, the peak intensity is held constant at $10 \mathrm{TW} / \mathrm{cm}^{2}$. White regions represent pulses that either produce negligible wave packet amplitude $\left(<1 \times 10^{-10} a_{0}\right)$ or are comprised of envelopes that are too narrow to allow for one full field oscillation. The dashed black line shows the location of the harmonic eigenfrequency $\omega_{0}$ of the system, and the dotted black line shows the location of $\omega_{0} / 2$. Note how the peaks present at these frequencies become narrower as the pulse width increases. The red line shows the location of the $1 \mathrm{D}$ cross section plotted in Fig. 2.

The peak of this function indicates that there is a pulse width that will produce maximum excitation. The location of this peak can be found by differentiating Eq. (23) and can be shown to be proportional to the system oscillation period $T=2 \pi / \omega_{0}$,

$$
\sigma_{\text {optimum }}=\frac{T}{\pi \sqrt{2}}
$$

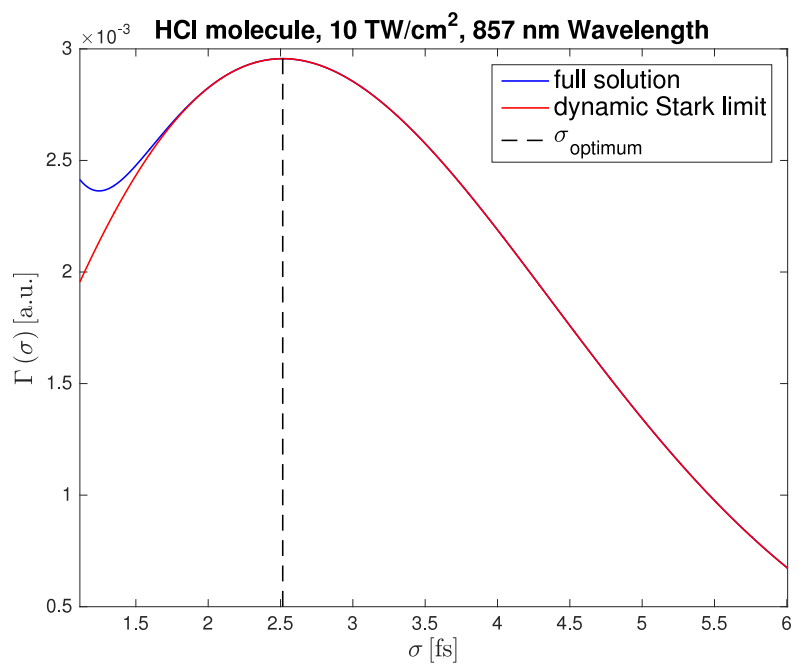

FIG. 2. The wave packet amplitude when the central frequency of the driving Gaussian pulse approaches the off-resonant Stark limit. As in Figure 1, the peak intensity is held fixed at $10 \mathrm{TW} / \mathrm{cm}^{2}$. In this regime, the system ceases to respond to the pulse frequency and instead the excitation becomes primarily dependent on the shape of the pulse envelope. The blue curve indicates the full solution from Eq. (20), the red curve shows the predicted behavior when the dynamic Stark effect is the only contributing factor to wave packet amplitude as per Equation (23), and the dashed line shows the location of optimal pulse width given by the relation in Eq. (24). 
Note that in Figs. 1 and 2, pulse fluence is not held constant since we vary the width and keep the peak intensity fixed. The motivation behind this choice is traced to the fact that at the frequencies we are considering, it can be shown that limiting the peak intensity is of critical importance when it comes to diminishing unwanted ionization effects, whereas changes in the pulse fluence have been shown to be less impactful in this respect. ${ }^{44-47}$ If we choose to change the parameters of our analysis by instead keeping the pulse energy constant, it would not be possible to calculate a non-trivial optimal pulse width like the one in Eq. (24). The reason for this can easily be understood considering that in order to keep the fluence constant, the amplitude $A_{0}$ must be inversely proportional to the square root of the pulse width, i.e., $A_{0} \propto 1 / \sqrt{\sigma}$. Inserting this relation into Eq. (23), we see that the exponential prefactor will become independent of $\sigma$, resulting in a monotonic increase in the wave packet amplitude as $\sigma \rightarrow 0$.

We will now demonstrate the impact of applying a pure Gaussian envelope to our model in the dynamic Stark limit. Defining the envelope function as

$$
E_{\text {env }}(t)=A_{0} \exp \left(-\frac{t^{2}}{2 \sigma^{2}}\right),
$$

we see that setting $\omega_{c}=0$ in Eq. (19) will result in the same expression. We can use this relation to immediately find the solutions to Eq. (15) when $F(T)=E_{\text {env }}(t)$ by simply setting $\omega_{c}=0$ in the results we have already derived. The amplitude that arises due to coupling with the polarizability term can be calculated in this way using Eq. (22), and is found to be

$$
I_{\alpha}\left(A_{0}, \omega_{c}, \sigma\right)_{\omega_{c}=0}=\frac{\alpha^{\prime} A_{0}^{2} \sigma \sqrt{\pi}}{2 \omega_{0} m} \exp \left(-\frac{\sigma^{2} \omega_{0}^{2}}{4}\right) .
$$

Note that setting $\omega_{c}=0$ is just a mathematical trick, and for the purposes of this derivation, we can assume the system is still operating in the dynamic Stark regime. This allows us to disregard contributions from the permanent molecular dipole term, meaning Eq. (26) is an expression for the total wave packet amplitude in this limit, i.e.,

$$
\Gamma\left(A_{0}, \sigma\right)_{\mathrm{env}}=I_{\alpha}\left(A_{0}, \omega_{c}, \sigma\right)_{\omega_{c}=0} .
$$

Comparing Eqs. (26), (27), and (23), we see that $\Gamma\left(A_{0}, \sigma\right)_{\mathrm{env}}$ $=2 \times \Gamma\left(A_{0}, \sigma\right)_{\left|\omega_{c}\right| \gg \omega_{0}}$. This demonstrates how contributions to the wave packet amplitude from the molecular polarizability term will double when the carrier frequency is eliminated from the pulse envelope. The reason for this is obvious when one considers that as a potential surface is shifted by a field oscillating in the $\left|\omega_{c}\right| \gg \omega_{0}$ limit, the wave packet will only "feel" the period averaged displacement since it cannot react quickly enough to the rapidly changing field components. Since the contribution from the molecular polarizability term is proportional to the square of the driving pulse, this is equivalent to multiplying the squared envelope function by a factor of $1 / 2$, the upshot of which is that in the dynamic Stark limit, Eq. (14) can be simplified into the familiar form: ${ }^{31}$

$$
V(R, t)=\frac{1}{2} m \omega_{0}^{2} R^{2}-\frac{1}{4} \alpha^{\prime} E_{\mathrm{env}}^{2}(t) R,
$$

where the last term is equivalent to a time-dependent shift of the potential following the squared pulse envelope.

\section{A. Pulse trains}

We now investigate the impact of splitting the Gaussian pulse described by Eq. (19) into a train of $N$ identical copies of itself separated by time interval $\tau$, making use of the linearly forced harmonic oscillator model to derive analytical solutions to the time-dependent dynamics. We assume the pulses interact with the molecule in the dynamic Stark limit, and all share the same phase (i.e., they constructively interfere). The envelope of the pulse train function can therefore be expressed as

$$
E_{\text {env }}(t)=\frac{A_{0}}{\sqrt{N}} \sum_{n=0}^{N-1} \exp \left(-\frac{(t-n \tau)^{2}}{2 \sigma^{2}}\right) .
$$

Note that the peak amplitude of each pulse in the series is set to $A_{0} / \sqrt{N}$, which ensures that the total fluence of the pulse train will be equivalent to that of the original pulse. Inserting (29) into Eq. (15) and solving in the dynamic Stark limit, the general solution for the wave packet amplitude is found to be

$$
\begin{aligned}
\Gamma\left(A_{0}, \sigma, \tau, N\right)= & \frac{\alpha^{\prime} A_{0}^{2} \sigma \sqrt{\pi}}{4 \omega_{0} m} \exp \left(-\frac{\sigma^{2} \omega_{0}^{2}}{4}\right) \\
& \times \frac{1}{N}\left[N+\sum_{n \neq m}^{N-1} \cos \left(\omega_{0} \tau[n-m]\right)\right]^{1 / 2} .
\end{aligned}
$$

If $\tau=2 \pi k / \omega_{0}=k T$, where $k \in \mathbb{N}$ and $T$ is the length of one wave packet oscillation period, the sum in the brackets of Eq. (30) can be rewritten as

$$
\sum_{n \neq m}^{N-1} \cos (2 \pi k[n-m])=N(N-1) .
$$

Combining relations (30) and (31), we see that the second line of Eq. (30) becomes unity and the functional dependency on $N$ and $\tau$ drops out, leaving a result that is identical to Eq. (23). This means that when the pulse spacing $\tau$ is an integer multiple of the system oscillation period $T$, the wave packet amplitude induced by the pulse train is equivalent to the amplitude produced by a single pulse of equivalent energy. This indicates that when they are spaced in this manner, the effect of each sub-pulse on the total amplitude will be additive. It is interesting to note that these results will also hold when the pulses in the train significantly overlap.

If we instead consider a pulse train containing only two pulses, and let $\tau=T(k+1 / 2)$, i.e., the spacing between the pulses is an integer number of wave packet oscillation periods plus $1 / 2$, we find that the bracketed term in Eq. (30) disappears, since

$$
\sum_{n \neq m}^{N-1} \cos (2 \pi(k+1 / 2)[n-m])=-2,
$$

when $N=2$ and $k \in \mathbb{N}$. This result demonstrates that spacing two pulses in this manner will lead to no net excitation of the system, since effectively the oscillations set in motion by the first pulse are exactly canceled by the second pulse. In this way, we can see that the effect of multiple pulses on the wave packet amplitude may also be subtractive, depending on how we time their arrival to the system. Here, one may consider the analogy of pushing a child on a swing, since it is clear that 
the change in the swing amplitude depends largely on when we apply our push.

If $\tau \neq k T$ and $N$ is very large, it can be shown that the sum term in Eq. (30) will be $\ll N$, since the negative and positive contributions to the sum will on average cancel each other out. In this limit, the resulting amplitude will be negligible since the solution given by Eq. (30) will be proportional to $1 / \sqrt{N}$. In this way it is shown how the ability to control the spacing in a train of pulse envelopes can effectively be used to highlight certain system transitions by dampening others. Note, also, that using a pulse train in place of a single pulse allows us to limit the peak intensity while still exerting control over the molecule, an important factor when it comes to minimizing unwanted ionization.

In 1991, it was demonstrated how individual phonon modes in an $\alpha$-perylene molecular crystal sample were excited by trains of pulses with non-resonant carrier frequencies when the inter-pulse spacing was equivalent to a modal oscillation period..$^{33}$ It was also found that applying a single pulse of equivalent energy to the same system resulted in the excitation of a large number of different modes, i.e., the ability to selectively excite an individual mode was lost. These experimental results are in accordance with our theoretical predictions that controlling the pulse train spacing leads to modal selectivity.

More recently, it has been shown that the amplitude of the induced dihedral oscillations in a molecule approximately doubled when the system was hit with two Gaussian kick pulses instead of one, provided the temporal separation between the pulses was equivalent to the system oscillation period. It was also shown that the dihedral oscillations became very small when the spacing between the pulses was reduced to roughly half a system oscillation period. ${ }^{20}$ This backs up our result showing that the effect of multiple pulses on the wave packet oscillation amplitude can be additive or subtractive, depending on how we adjust the timing between each pulse.

\section{B. Frequency-space interpretation}

Assuming conditions where the dynamic Stark effect dominates and the system response is independent of the pulse frequency, and given a generalized pulse envelope function $E_{\text {env }}(t)$, we can use Eqs. (15), (18), and (20) to show that the wave packet amplitude when $t \rightarrow \infty$ can be written as

$$
\begin{aligned}
\Gamma\left(A_{0}, \sigma\right)= & \frac{\alpha^{\prime}}{4 \omega_{0} m}\left(\left[\int_{-\infty}^{\infty} \cos \left(\omega_{0} t\right) E_{\mathrm{env}}^{2}(t) d t\right]^{2}\right. \\
& \left.+\left[\int_{-\infty}^{\infty} \sin \left(\omega_{0} t\right) E_{\mathrm{env}}^{2}(t) d t\right]^{2}\right)^{1 / 2} .
\end{aligned}
$$

If $E_{\text {env }}(t)$ is a square integrable function, Parseval's theorem ${ }^{48}$ allows us to recast Eq. (33) in the spectral domain

$$
\begin{aligned}
\Gamma\left(A_{0}, \sigma\right)= & \frac{\alpha^{\prime}}{8 \pi \omega_{0} m}\left(\left[\int_{-\infty}^{\infty} \tilde{E}_{\mathrm{env}}(\omega) \tilde{E}_{\mathrm{env}}^{*}\left(\omega-\omega_{0}\right) d \omega\right]\right. \\
& \left.\times\left[\int_{-\infty}^{\infty} \tilde{E}_{\mathrm{env}}(\omega) \tilde{E}_{\mathrm{env}}^{*}\left(\omega+\omega_{0}\right) d \omega\right]\right)^{1 / 2},
\end{aligned}
$$

where $\tilde{E}_{\text {env }}(\omega)$ is the Fourier transform of $E_{\text {env }}(t)$ and the * superscript denotes complex conjugation. Inspecting the two integrals on the right side of Eq. (34) shows that the width of the spectral distribution must be broader than $\omega_{0}$ in order to lead to appreciable vibrational amplitude. Additionally, the form of Eq. (34) can generate insight as to how the underlying mechanism of the dynamic Stark effect can be interpreted as impulsive stimulated Raman scattering. To understand why this is, consider first the schematic shown in Fig. 3(a) of a Stokes-Raman scattering event, in which the ground state of a harmonic oscillator is excited up one level via a two step process consisting of transitions to and from a higher lying virtual state by two non-resonant frequencies.

This type of transition may occur provided that the spectral distribution of the laser pulse contains at least two frequencies where the difference between these frequencies is an eigenfrequency of the system. To further illustrate the

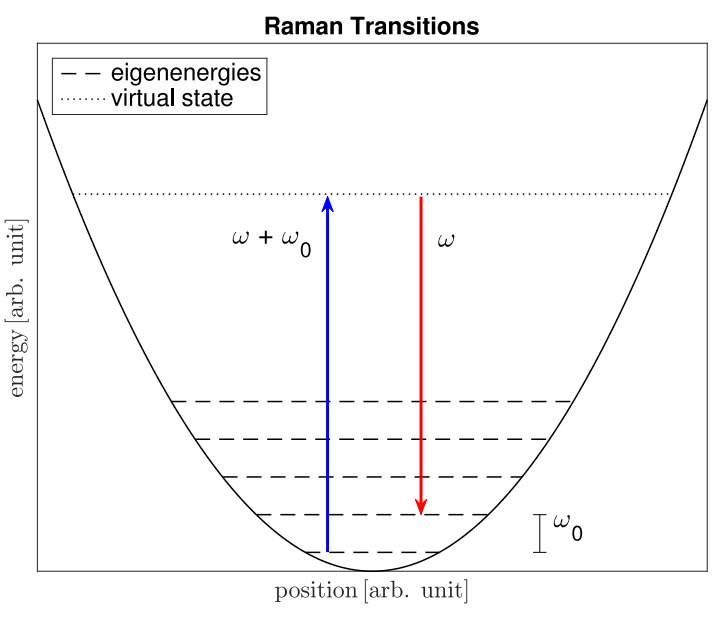

(a)

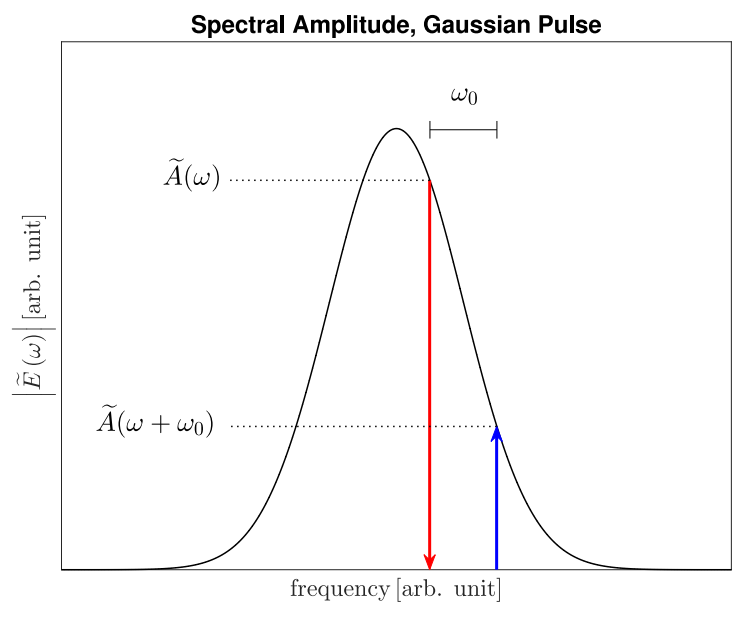

(b)

FIG. 3. (a) A pictorial representation of a Stokes-Raman scattering event in the quantum harmonic oscillator. Starting in the ground state, a photon with energy $\hbar\left(\omega+\omega_{0}\right)$ is absorbed, resulting in a transition to a higher virtual state. Strictly speaking, this is a forbidden transition; however, emission of a photon with energy $\hbar \omega$ directly afterwards results in a net absorption of $\hbar \omega_{0}$. (b) Spectral amplitude of a temporal Gaussian pulse. The arrows indicate the location of one possible Stokes-Raman scattering event. To find the total probability of a certain transition taking place, we must integrate the probability of all possible scattering events of a specific frequency, as indicated by Eq. (34). 
point, assume that the transitions illustrated in Figure 3(a) are caused by a flat phase spectral distribution of a Gaussian pulse like the one from Eq. (19), as shown in Fig. 3(b). Here we see that if the pulse contains frequencies separated by the harmonic eigenfrequency $\omega_{0}$, it may generate transitions to and from higher lying virtual states and thereby produce non-resonant transitions in our model system.

To show how this picture relates to Eq. (34), we put forth the naive argument that the probability of the transition in Fig. 3(b) taking place must somehow be proportional to the product of the spectral amplitudes of the two frequency components $\tilde{A}(\omega)$ and $\tilde{A}\left(\omega+\omega_{0}\right)$,

$$
P_{\uparrow \omega \downarrow \omega+\omega_{0}} \propto \tilde{A}(\omega) \tilde{A}\left(\omega+\omega_{0}\right) .
$$

Building on this notion, the total probability of a Raman transition of a specific frequency taking place must be proportional to the sum of all possible two step transitions that the frequency content of the pulse will allow, which can be expressed in integral form as

$$
P_{\text {tot }} \propto \int_{-\infty}^{\infty} \tilde{A}(\omega) \tilde{A}\left(\omega+\omega_{0}\right) d \omega .
$$

Comparing Eqs. (36) and (34) we see that they are qualitatively similar. Indeed, for a flat phase spectral distribution, i.e., given $\tilde{E}_{\text {env }}(\omega)=\left|\tilde{E}_{\text {env }}(\omega)\right| \exp (i \phi(\omega))$ and $\phi(\omega)=$ const., it can easily be shown that $\int_{-\infty}^{\infty} \tilde{E}_{\text {env }}(\omega) \tilde{E}_{\text {env }}^{*}\left(\omega+\omega_{0}\right) d \omega$ $=\int_{-\infty}^{\infty} \tilde{E}_{\text {env }}(\omega) \tilde{E}_{\text {env }}^{*}\left(\omega-\omega_{0}\right) d \omega$, in which case the two expressions are identical save for the fact that in the exact solution given by Eq. (34) we have been able to determine the pre-factor as well.

These results, particularly the form of Eq. (36), are quite similar to results obtained by Meshulach and Silberberg. ${ }^{36}$ The key difference is that in our paper, Eq. (34) is in principle exact, although our model of vibrational excitation is a simplified one. Qualitatively, the work done by Meshulach and Silberberg takes the opposite approach by invoking an approximate solution to a fully described system, it is therefore noteworthy that they arrive at an expression similar to Eq. (36).

We can exploit our understanding of the mechanics behind the dynamic Stark effect to construct so-called dark pulses, ${ }^{36}$ i.e., pulses with envelope shapes designed to make Raman transitions impossible within our model system of the linearly forced harmonic oscillator. We have already considered one example of a dark pulse envelope in this paper, namely, the case where a system with oscillation period $T$ is driven by two sub-pulses with a temporal separation of $T(k+1 / 2), k \in \mathbb{N}$. While we demonstrated it analytically for completeness, from a temporal perspective it is perhaps fairly intuitive why this kind of pulse will produce no excitation.

A less trivial way we can construct a dark pulse is by using our knowledge of the relationship between the spectral distribution of the pulse envelope and the Raman transition rate. Equation (34) indicates that the integrand functions are effectively multiplied by -1 any time the phases of $\tilde{E}_{\text {env }}(\omega)$ and $\tilde{E}_{\text {env }}\left(\omega \pm \omega_{0}\right)$ differ by $n \pi$ (where $n \in \mathbb{Z}$ ). Therefore, phase-chirping $\tilde{E}_{\text {env }}(\omega)$ with a $\pi$ step function over certain intervals can be shown to either limit or fully extinguish the rate of Raman transitions.

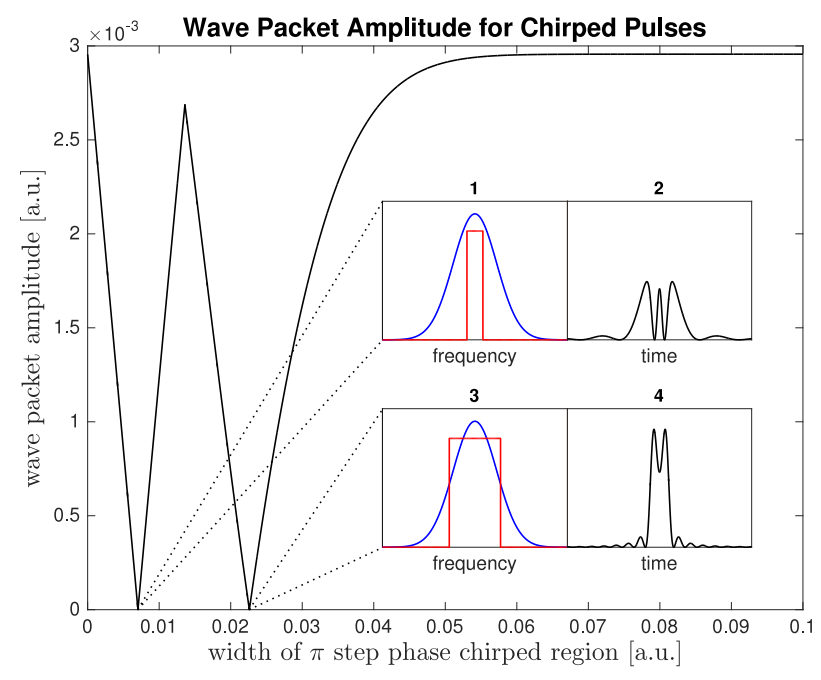

FIG. 4. This plot shows how the wave packet amplitude $\Gamma$ changes as the width of the $\pi$ step boxcar phase function increases. Insets 1 and 3 show sketches of the spectral pulse amplitude (blue) and the $\pi$ step phase functions (red) when $\Gamma=0$, while insets 2 and 4 show the corresponding temporal dark pulse envelope intensities $\propto\left|E_{\text {env }}(t)\right|^{2}$.

To exemplify this idea, we choose $\tilde{E}_{\text {env }}(\omega)$ to be the spectral distribution of a Gaussian pulse with $\sigma=\sigma_{\text {optimum }}$ as per Eq. (24). Figure 4 shows the analytic solution to Eq. (34) when $\tilde{E}_{\text {env }}(\omega)$ is phase chirped by a variable width $\pi$ step "boxcar" function centered at the spectral intensity peak.

As we would expect, when the chirped region becomes very wide, $\Gamma$ approaches the same value as that of the un-chirped pulse. The two minima where the wave packet amplitude becomes 0 occur when the width of the $\pi$ chirped region is such that the positive and negative contributions to the integrals in Eq. (34) cancel each other out. The envelope intensities of these two dark pulses are sketched in insets 2 and 4 of Fig. 4, and their corresponding spectral amplitudes and phase functions are shown in insets 1 and 3. From a purely temporal perspective, it is not at all obvious that these envelope shapes should both be dark pulses, illustrating how knowledge of the spectral mechanism behind the dynamic Stark effect can be a useful tool when it comes to predicting and/or analyzing how a pulse will interact with a molecular system.

\section{STATE-TO-STATE VIBRATIONAL EXCITATION VIA DSC-A NUMERICAL EXAMPLE}

While the analysis of simple pulse trains interacting with a harmonic potential allowed us to obtain analytic solutions to the time-dependent dynamics, so far we have only been able to affect the wave packet amplitude, due to the Ehrenfest (classical) dynamics of our simplified harmonic model.

We will now consider a more realistic system which will allow us to capture more complex behavior. The purpose of this investigation is twofold: One goal is to examine how well the principles discussed in Sec. III will generalize when we move beyond the harmonic approximation. Additionally, we feel it may be informative to demonstrate, as a proof of principle, that DSC can be employed to control dynamics that 
are more sophisticated than simply increasing or decreasing the wave packet amplitude.

To this end, we expand the model of the $\mathrm{HCl}$ molecule to a Morse potential. As in the harmonic approximation, we retain the $1 \mathrm{D}$ description by once again assuming that the molecule is aligned with the field polarization direction along the $z$ axis. The polarizability function will now be a semi-empirical function, ${ }^{42}$ instead of the linear expansion we have been using thus far. Once again we assume the pulse carrier frequency is non-resonant and disregard any contributions to the molecular energy from the permanent electric dipole function. The pulse is simulated interacting with the $\mathrm{HCl}$ molecule using the split-operator method ${ }^{49}$ to solve the time-dependent Schrödinger equation, where the time-dependent part of Eq. (12) is modeled by the simplified expression that holds in the dynamic Stark limit,

$$
\hat{C}_{\text {int }}(R, t)=\frac{1}{4} \alpha(R) E_{\text {env }}^{2}(t) .
$$

With respect to our stated intentions, the objective of achieving a state-to-state transition within this model was determined to be a suitable goal, as modifying the shape of the wave packet to overlap a target eigenstate calls for a more refined approach to the distribution of pulse energy over time than we have considered thus far. For this purpose, a genetic algorithm ${ }^{10}$ was used to optimize the envelope shape of a $1 \mathrm{ps}$ pulse, with the goal of maximizing the population transfer from the ground to the second vibrational state of the $\mathrm{HCl}$ molecule. The temporal pulse envelope is parameterized using a series of discrete, equally spaced regions or "bins." Each bin is assigned a constant value between 0 and some user-defined maximum that serves as the intensity cutoff limit. Construction of the actual pulse envelope for use in the simulation is accomplished by smoothing the entire series of bins using a Gaussian low pass filter, as shown in Fig. 5.

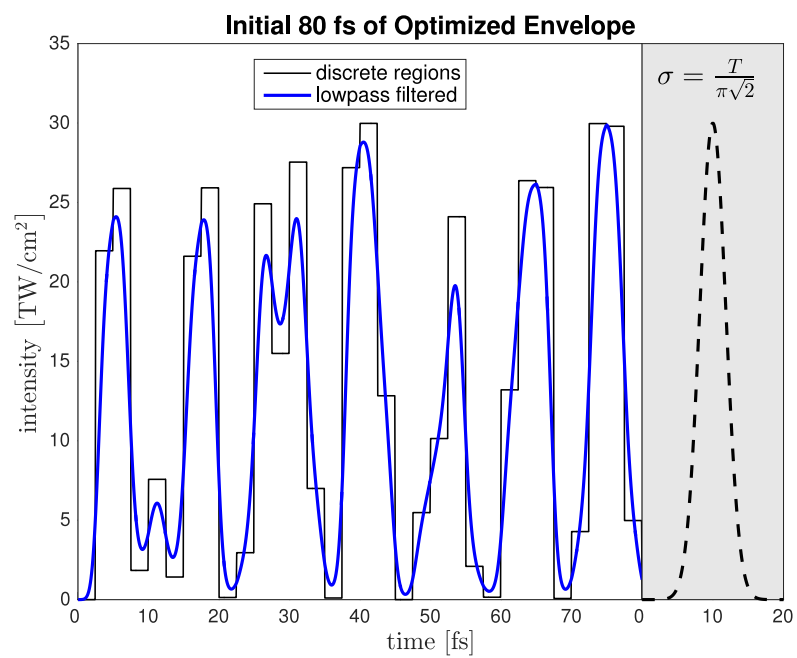

FIG. 5. The first 80 fs of the optimized pulse envelope shown in Fig. 6 (blue curve). The black line shows the pre-filtered distribution of the 2.5 fs discrete regions, where the genetic algorithm treats the height of each region as a free parameter. For comparison, the intensity-limited Gaussian pulse envelope calculated to maximize the wave packet amplitude within the harmonic approximation of the $\mathrm{HCl}$ model, in the dynamic Stark regime (as per Eq. (24)), is sketched on the same time scale in the gray box to the right.

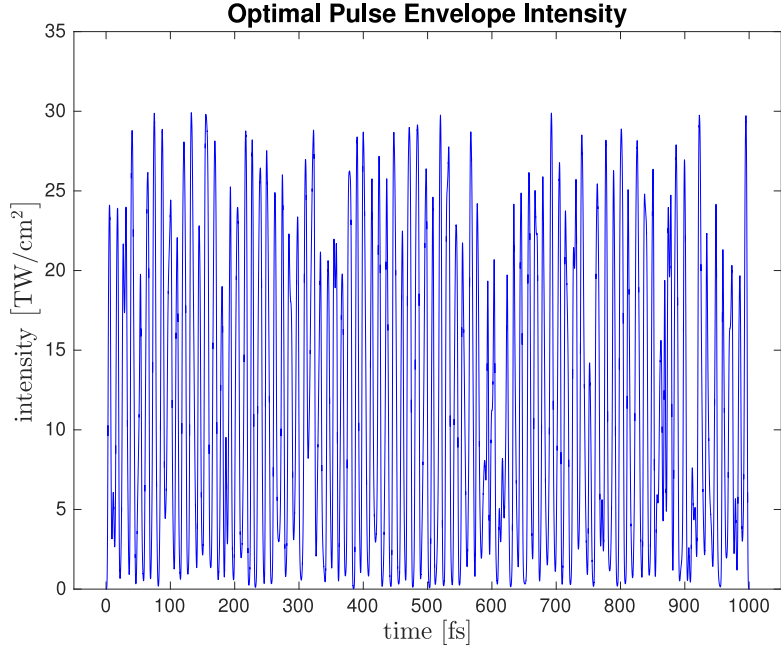

FIG. 6. The temporal intensity profile of the optimized pulse envelope. Note that the pulse carrier frequency (not pictured) is assumed to be oscillating much faster than the temporal evolution of the envelope shape.

The width of each bin was set to 2.5 fs, leading to a total of 400 free parameters distributed over the $1 \mathrm{ps}$ time window. The temporal standard deviation of the Gaussian smoothing kernel was set to $1 \mathrm{fs}$, and the intensity limit of the pulse envelope was set to $30 \mathrm{TW} / \mathrm{cm}^{2}$, which was estimated to be low enough as to not cause any appreciable system ionization using the FC-ADK tunneling ionization model. ${ }^{47}$ Using these parameters, the genetic algorithm was able to achieve $\sim 99 \%$ population transfer from the ground to second vibrational state of the $\mathrm{HCl}$ molecule after 346 generations with a population size of 2000 individuals. The full intensity profile of the optimized pulse envelope is shown in Fig. 6, and the occupancies of the first four vibrational states as a function of time are shown in Fig. 7.

The absolute value of the pulse envelope Fourier transform is shown in Fig. 8. For reference, we include the $0-1$ and 1-2 transition frequencies here. The spectral interpretation of Stokes-Raman scattering events which we

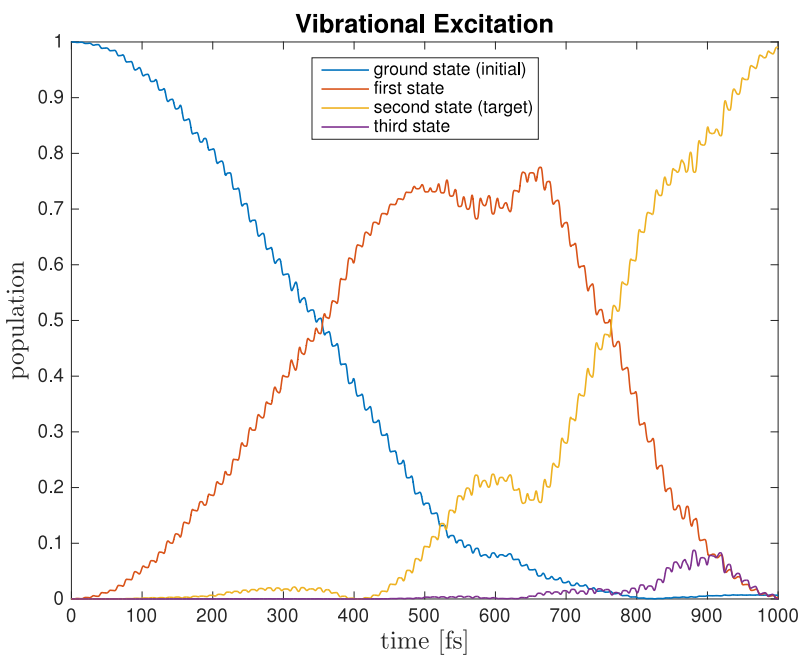

FIG. 7. Population of the ground, first, second, and third vibrational states of the $\mathrm{HCl}$ molecule when driven by the optimized pulse shown in Fig. 6, as a function of time. The final population of the second excited state is $>99 \%$. 


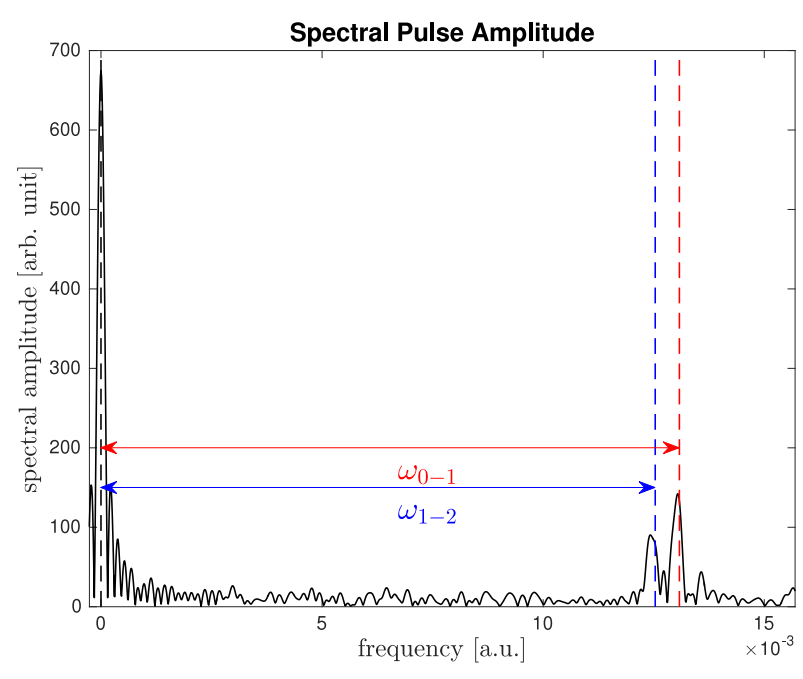

FIG. 8. Fourier transform of the optimized pulse envelope shown in Figure 6. Note that the pulse carrier frequency is disregarded here as well. Including the carrier frequency would merely shift the spectral distribution, but would not change its overall shape.

outlined in Eqs. (34) and (36) indicates that the optimized pulse should generate non-resonant transitions at the first two eigenfrequencies of the $\mathrm{HCl}$ molecule, since the spacing between the first and third peaks is very close to the $0-1$ transition frequency, and likewise the spacing between the first and second peaks is close to the 1-2 transition.

As Figs. 5 and 6 indicate, the pulse envelope required to achieve the population transfer exhibits a complicated pulse-train structure, containing a large number of sub-pulses that evolve on $\sim 5-7$ fs time scales. The sketch on the right side of Fig. 5 demonstrates how the sub-pulses present within the initial $80 \mathrm{fs}$ of the optimized envelope evolve on comparable time scales to that of the optimal Gaussian pulse envelope calculated using the harmonic model of $\mathrm{HCl}$ as discussed in Section III, and in particular, as expressed by Eq. (24). While the comparison is rough, it does make sense from a controllability perspective that the numerically optimized pulse would generally contain subfeatures with shapes similar to the analytically calculated result from Eq. (24).

The narrow temporal widths of the sub-pulses, which are a reflection of the high vibrational frequency of the $\mathrm{HCl}$ molecule, may be challenging to implement experimentally. Heavier molecules are attractive in this respect, since they generally vibrate on slower time scales. The degree of influence each sub-pulse has on the system is related to the magnitude of the first derivative of the polarizability function $\alpha(\mathbf{R})$. This means that systems where the polarizability changes significantly as a function of the relative atomic displacement(s) are particularly well suited for the application of DSC.

Generally, the production of non-trivial shaped laser pulses on ultra-fast time scales is accomplished by manipulating the spectral components of an unshaped laser pulse using a spectral pulse shaper. Using such a device, the frequency components of the unshaped pulse may be acted on by attenuating their intensity and/or modifying their phase. The ability to change the phase and intensity distributions of the unshaped spectral pulse allows for a large degree of controllability with respect to achieving a desired target pulse, however the cost of this approach is often a significant loss of energy due to the attenuation of the spectral components.

Phase-only pulse shaping is an attractive alternative in this respect, as energy losses are kept to a minimum. The sacrifice of a phase-only approach is that it becomes much harder, and in many cases impossible, to achieve an arbitrary target pulse envelope and carrier frequency distribution. Working with pulses that interact with molecules in the dynamic Stark limit allows us to disregard the carrier frequency and focus only on the pulse envelope. This relaxes the requirements on the target pulse and might open up the possibilities of generating arbitrary envelope shapes using phase-only spectral manipulation. While the task of determining the spectral phase function that will produce a desired envelope shape is still not trivial, there are a variety of iterative optimization methods which have been shown to be well suited for this task. ${ }^{50-52}$

As stated in the article outset, applying tailored laser pulses to control molecular transitions has been the subject of numerous studies. In particular, analytical insights have been developed for generating pulse shapes tuned to produce specific state-to-state vibrational transitions via coupling to the permanent molecular dipole moment. ${ }^{53,54}$ The observant reader may therefore wonder what the advantage of a pure DSC approach may be, when compared to these more established methods.

The answer comes down to the unique dynamics that apply in the regime of pure DSC. The fact that the dynamic Stark effect dominates under non-resonant conditions means that we can effectively disregard the carrier frequency of the laser, which is attractive from the standpoint of experimental implementation. Another obvious advantage of DSC compared to resonant approaches is that it can applied to molecules where there is no coupling to the permanent electric dipole moment.

DSC may be accomplished using "pseudo-frequencies," generated via pulse trains with spacings tuned to specific molecular modes. This approach is particularly interesting with respect to heavy molecules with long vibrational periods, as their low frequencies will typically lie outside the operational bandwidths of conventional laser and pulse shaping technologies.

One major drawback of a pure DSC approach is that the required intensities are typically much higher than those needed for resonant control. As discussed in this article, this problem may be mitigated by splitting high intensity pulses into trains of smaller sub-pulses; however this typically results in an increase of the overall temporal duration of the pulse envelope, which may in turn lead to issues with dissipation or other unwanted coupling effects.

\section{CONCLUDING REMARKS}

We have used the linearly forced harmonic oscillator to model the interaction of a laser pulse with a diatomic molecule which allows for analytic solutions to the time-dependent 
evolution of the quantum wave packet. Using these solutions, we have been able to show how the dynamic Stark effect arises from the interaction with the electric field envelope of a laser pulse, and an optimal pulse width has been determined.

The impact of driving our model with multiple evenly spaced pulses demonstrates how modal selectivity can be achieved. Additionally, we show that the effect of multiple pulses can contribute both additively and subtractively to the wave packet amplitude in our harmonic model.

Using Parseval's theorem, we describe and predict how a pulse interacts with our model system in the DSC limit using the spectral phase and amplitude of the pulse envelope. The spectral interpretation of DSC can generate insight that would not be available from a purely temporal perspective, which we demonstrate via phase-only manipulation of a flat-phase Gaussian to generate non-trivial dark pulse envelopes that produce no net excitation.

Finally, we demonstrate how quantum control may be achieved via the dynamic Stark effect by shaping a parameterized pulse envelope function, allowing us to produce state-to-state vibrational excitation in an anharmonic oscillator. The fact that the carrier frequency of the optimized envelope can be disregarded opens the door for arbitrary waveform generation by optical pulse shapers. We expect that careful pulse optimization will allow for dynamic Stark control of various more complicated competing processes which are currently being studied.

${ }^{1}$ S. A. Rice and M. Zhao, Optical Control of Molecular Dynamics (Wiley, New York, 2000).

${ }^{2}$ C. Brif, R. Chakrabarti, and H. Rabitz, New J. Phys. 12, 075008 (2010).

${ }^{3}$ M. Shapiro and P. Brumer, Quantum Control of Molecular Processes (Wiley, New York, 2012).

${ }^{4}$ A. H. Zewail, Phys. Today 33(11), 27 (1980).

${ }^{5}$ T. C. Weinacht, J. Ahn, and P. H. Bucksbaum, Nature 397, 233 (1999).

${ }^{6}$ I. Barth and J. Manz, Angew. Chem., Int. Ed. 45, 2962 (2006).

${ }^{7}$ R. S. Judson and H. Rabitz, Phys. Rev. Lett. 68, 1500 (1992).

${ }^{8}$ A. M. Weiner, Rev. Sci. Instrum. 98, 1929 (2000).

${ }^{9}$ A. Monmayrany, S. Weber, and B. Chatel, J. Phys. B 43, 103001 (2010).

${ }^{10}$ M. Mitchell, An Introduction to Genetic Algorithms, 5th ed. (MIT Press, 1999).

${ }^{11}$ A. Assion, T. Baumbert, M. Bergt, T. Brixner, B. Kiefer, V. Seyfried, M. Strehle, and G. Gerber, Science 282, 919 (1998).

${ }^{12}$ R. J. Levis, G. M. Menkir, and H. Rabitz, Science 292, 709 (2001).

${ }^{13}$ B. J. Pearson, J. L. White, T. C. Weinacht, and P. H. Bucksbaum, Phys. Rev. A 63, 063412 (2001).

${ }^{14}$ G. Vogt, G. Krampert, P. Nikaus, P. Nuernberger, and G. Gerber, Phys. Rev. Lett. 94, 068305 (2005).

${ }^{15}$ J. Savolainen, R. Fanciulli, N. Dijkhuizen, A. L. Moore, J. Hauer, T. Buckup, M. Motzkus, and J. L. Herek, Proc. Natl. Acad. Sci. U. S. A. 105, 7641 (2008).

${ }^{16}$ A. C. Florean, D. Cardoza, J. L. White, J. K. Lanyi, R. J. Sension, and P. H. Bucksbaum, Proc. Natl. Acad. Sci. U. S. A. 106, 10896 (2009).
${ }^{17}$ H. Stapelfeldt and T. Seideman, Rev. Mod. Phys. 75, 543 (2003).

${ }^{18}$ H. Niikura, P. B. Corkum, and D. M. Villeneuve, Phys. Rev. Lett. 90, 203601 (2003).

${ }^{19}$ C. B. Madsen, L. B. Madsen, S. S. Viftrup, M. P. Johansson, T. B. Poulsen, L. Holmegaard, V. Kumarappan, K. A. Jørgensen, and H. Stapelfeldt, J. Chem. Phys. 130, 234310 (2009).

${ }^{20}$ L. Christensen, J. H. Nielsen, C. B. Brandt, C. B. Madsen, L. B. Madsen, C. S. Slater, A. Lauer, M. Brouard, M. P. Johansson, B. Shepperson, and H. Stapelfeldt, Phys. Rev. Lett. 113, 073005 (2014).

${ }^{21}$ B. J. Sussman, D. Townsend, M. Y. Ivanov, and A. Stolow, Science 314, 278 (2006).

${ }^{22}$ D. Townsend, B. J. Sussman, and A. Stolow, J. Phys. Chem. A 115, 357 (2011).

${ }^{23}$ M. E. Corrales, J. González-Vázquez, G. Balerdi, I. R. Solá, R. de Nalda, and L. Bañares, Nat. Chem. 6, 785 (2014).

${ }^{24}$ I. R. Solá, J. González-Vázquez, R. de Nalda, and L. Bañares, Phys. Chem. Chem. Phys. 17, 13163 (2015).

${ }^{25}$ Q. Ren, G. G. Balint-Kurti, F. R. Manby, M. Artamonov, T.-S. Ho, and H. Rabitz, J. Chem. Phys. 124, 014111 (2006).

${ }^{26}$ J. Haruyama, C. Hu, and K. Watanabe, Phys. Rev. A 85, 062511 (2012).

${ }^{27}$ P. Atkins and R. Friedman, Molecular Quantum Mechanics, 4th ed. (Oxford University Press, 2005).

${ }^{28}$ S. Sharma, H. Singh, and G. G. Balint-Kurti, J. Chem. Phys. 132, 064108 (2010).

${ }^{29}$ C. Sanz-Sanz, G. W. Richings, and G. A. Worth, Faraday Discuss. 153, 275 (2011).

${ }^{30}$ Y. Korosaki, T.-S. Ho, and H. Rabitz, J. Chem. Phys. 140, 084305 (2014).

${ }^{31}$ B. J. Sussman, Am. J. Phys. 79, 477 (2011).

${ }^{32}$ D. J. Griffiths, Introduction to Quantum Mechanics, 2nd ed. (Pearson Education International, New Jersey, 2005).

${ }^{33}$ A. M. Wiener, D. E. Leaird, G. P. Wiederrecht, and K. A. Nelson, J. Opt. Soc. Am. B 8, 1264 (1991).

${ }^{34}$ R. Kosloff and A. D. Hammerich, Phys. Rev. Lett. 69, 2172 (1992).

${ }^{35}$ J. A. Cina and T. J. Smith, J. Chem. Phys. 98, 9211 (1993).

${ }^{36}$ D. Meshulach and Y. Silberberg, Phys. Rev. A 60, 1287 (1999).

${ }^{37}$ R. A. Bartels, S. Backus, M. M. Murnane, and H. C. Kapteyn, Chem. Phys. Lett. 374, 326 (2003).

${ }^{38}$ R. P. Feynman, Phys. Rev. 56, 340 (1939).

${ }^{39}$ N. E. Henriksen and F. Y. Hansen, Theories of Molecular Reaction Dynamics (Oxford University Press, 2008).

${ }^{40} \mathrm{D}$. J. Tannor, Introduction to Quantum Mechanics: A Time-Dependent Perspective (University Science Books, 2007).

${ }^{41}$ M. Machholm and N. E. Henriksen, J. Chem. Phys. 111, 3051 (1999).

${ }^{42}$ M. A. Buldakov, V. N. Cherepanov, and N. S. Nagornova, J. Comput. Methods Sci. Eng. 6, 153 (2006).

${ }^{43}$ J. F. Harrison, J. Chem. Phys. 128, 114320 (2008).

${ }^{44}$ A. M. Perelomov, V. S. Popov, and M. V. Terent'ev, J. Exp. Theor. Phys. 50, 1393 (1966).

${ }^{45}$ X. M. Tong, Z. X. Zhao, and C. D. Lin, Phys. Rev. A 66, 033402 (2002).

${ }^{46}$ E. Räsänen and L. B. Madsen, Phys. Rev. A 86, 033426 (2012).

${ }^{47}$ J. Förster, Y. V. Vanne, and A. Saenz, Phys. Rev. A 90, 053424 (2014).

${ }^{48}$ W. Rudin, Real and Complex Analysis, 3rd ed. (McGraw-Hill, 1987).

${ }^{49}$ R. Kosloff, J. Phys. Chem. 92, 2087 (1988).

${ }^{50}$ A. M. Weiner, S. Oudin, D. E. Leaird, and D. H. Reitze, J. Opt. Soc. Am. A 10, 1112 (1993).

${ }^{51}$ D. Meshulach, D. Yelin, and Y. Silberberg, J. Opt. Soc. Am. B 15, 1615 (1998).

${ }^{52}$ M. Hacker, G. Stobrawa, and T. Feurer, Opt. Express 9, 191 (2001).

${ }^{53}$ M. V. Korolkov and G. K. Paramonov, Phys. Rev. A 57, 4998 (1998).

${ }^{54}$ S. Chelkowski, A. D. Bandrauk, and P. B. Corkum, Phys. Rev. Lett. 65, 2355 (1990). 